\title{
Stable planes with a point transitive abelian group
}

\author{
Andreas Wörner*
}

\begin{abstract}
We consider stable planes $E=(P, \mathcal{L})$ admitting an abelian group $\Delta$ which acts (sharply) transitively on the point set $P$. Known examples are the so-called arc planes with $\Delta=\mathbb{R}^{2}$, see [1], affine translation planes and shift planes. We examine the possible actions of $\Delta$ on the line space $\mathcal{L}$ and use the results in order to characterize affine shift planes and translation planes.
\end{abstract}

Keywords : stable planes, abelian group action, arc planes

MSC 2000: 51H10

\section{Introduction}

Let $E=(P, \mathcal{L})$ be a stable plane with $P$ locally compact, connected and of positive finite dimension. This implies, according to [5] (1.11) and [7] Theorem 1, that each line has dimension $l$ and $P$ has dimension $2 l$. Throughout this article we assume that the automorphism group $\Sigma$ of the stable plane $E$ contains an abelian subgroup $\Delta \leq \Sigma$ which acts transitively (and hence sharply transitively) on the point space $P$.

Well-known affine examples are translation planes and shift planes (that is, planes with point set $\mathbb{R}^{2 l}$ and lines of the form $\{c\} \times \mathbb{R}^{l}, c \in \mathbb{R}^{l}$, plus all translates of a graph of a suitable function $f: \mathbb{R}^{l} \rightarrow \mathbb{R}^{l}$ ). We prove that there are no other affine planes satisfying our assumptions. In particular, if $E$ is affine, $P$ is isomorphic to $\mathbb{R}^{2 l}$ and each line is homeomorphic to $\mathbb{R}^{l}$. For $l=2$ this agrees with

\footnotetext{
${ }^{*}$ The author thanks Rainer Löwen for his numerous advice in writing papers.
} 




\section{Now we prove Theorem 2.4.}

Proof of Theorem 2.4. Assume, by way of contradiction, that all lines are arcs. Let $\left\{L_{i} \mid i \in I\right\}$ be a representing system of orbits and $p \in P$. According to Lemma 2.5, each set $L_{i}^{\Delta} \cap \mathcal{L}_{p}$ is open in $\mathcal{L}_{p}$. The union of these sets is a disjoint open cover of $\mathcal{L}_{p}$, because there are no straight lines. Since $\mathcal{L}_{p}$ is connected according to $[5,(1.14)]$, it follows that $|I|=1$; so there is only one line orbit, say $L^{\Delta}=\mathcal{L}$. According to Lemma 2.5, we have $L \approx L^{\Delta} \cap \mathcal{L}_{p}=\mathcal{L}_{p}$. Since $\mathcal{L}_{p}$ is compact according to [2, (3.8)], the line $L$ and hence each line is compact. Now [2, (3.10)] and (3.11) show that $E$ is a projective plane and the point space $P$ is compact. Thus, $\Delta$ is a compact connected abelian Lie group and therefore (see e.g. [10, (94.38)]) a torus. In particular, the fundamental group of $P$ is isomorphic to $\mathbb{Z}^{2 l}$, in contradiction to [10, (51.28)] for $l \geq 2$ or to [10, (52.14)] for $l=1$.

The presence of straight lines in our stable plane allows us to prove that all lines are manifolds. As a first step we have the following lemma.

Lemma 2.6. Let $K$ be a straight line. Then $K$ is a manifold and the Lie group $\Delta_{K}$ acts sharply transitively on $K$.

Proof. Let $o \in K$. By definition we have $o^{\Delta_{K}} \subseteq K$. Assume, by way of contradiction, that there is a point $p \in K \backslash o^{\Delta_{K}}$, say $p=o^{\delta}$ with $\delta \in \Delta \backslash \Delta_{K}$. Since $\Delta$ is abelian and $p \in K$, we obtain that $K \supseteq p^{\Delta_{K}}=o^{\delta \cdot \Delta_{K}}=o^{\Delta_{K} \cdot \delta}$. Hence $\left(o^{\Delta_{K}}\right)^{\delta} \subseteq K \cap K^{\delta}$, and $K=K^{\delta}$ since $\left|o^{\Delta_{K}}\right| \geq 2$. This contradicts $\delta \notin \Delta_{K}$.

We have shown that $\Delta_{K}$ is (sharply) transitive on $K$. The mapping $\Psi_{o}: \Delta \rightarrow$ $P$ (defined as in the proof of Proposition 2.2) restricts to a homeomorphism $\Delta_{K} \approx K$. Since $K \subseteq P$ is closed $([5,(1.3)])$, the stabilizer $\Delta_{K}$ is a closed subgroup of the Lie group $\Delta$, hence a Lie group. In particular, $\Delta_{K}$ and $K$ are manifolds.

As an immediate consequence we can prove for later use

Lemma 2.7. For a straight line $K$ and $p \in P$ we have that $\left|K^{\Delta} \cap \mathcal{L}_{p}\right|=1$.

Proof. Without loss of generality we may assume that $p \in K$. Let $\delta \in \Delta$, then

$$
K^{\delta} \in \mathcal{L}_{p} \Longleftrightarrow \exists k \in K: k^{\delta}=p \Longleftrightarrow \delta \in \Delta_{K},
$$

where the equivalence on the right hand side follows from Lemma 2.6 (existence of a $\gamma \in \Delta_{K}$ with $k^{\gamma}=p$ ) together with the sharply transitive action of $\Delta$ (uniqueness of this $\gamma$ ). Hence $K^{\delta}=K$, which proves the assertion. 




